\title{
Adult mental health disorders and their age at onset
}

P. B. Jones

\begin{abstract}
Summary
The study of age at onset of mental health disorders is technically and conceptually difficult. It is important to consider these age distributions in order to understand causes and mechanisms of illness and to intervene at an appropriate juncture for primary and secondary prevention. This article reviews some of the approaches to studying age at onset, sets out the evidence to support the assertion that adult mental disorders begin in adolescence, and finds that perhaps half of all adult mental health disorders have begun by the teenage years. The paper then discusses whether this
\end{abstract}

fits what is known about the developmental neurobiology of the brain and introduces the implications for mental health services.

\section{Declaration of interest}

P.B.J. nominated his department to receive a fee for providing scientific advice to Roche. He receives honoraria for contributing to the biannual British Association of Psychopharmacology schizophrenia master class series.
Adult mental health disorders begin by adolescence. This seems a simple enough statement that suggests we cannot understand mental illness affecting adults unless we appreciate what goes on during the preceding period of life. If true, childhood and adolescence, divided by the biological gateway of puberty, would be crucial epochs in terms of understanding causes and mechanisms of adult illness. This would frame attempts at early intervention and both primary and secondary prevention. Following the theme of this supplement, this paper focuses on the continuities between the adolescent and early adult stages of the life course, arguing that these are better seen as a single developmental epoch. However, the seeds of many conditions manifest in this period are sown in even earlier life, such that some reference is also made to childhood.

In some conditions such as psychotic illness, intervention during incipient illness or its early stages may prevent a lifetime of disability for a proportion of those affected. ${ }^{1}$ Onset of the full syndrome at a very young age is often associated with a severe course, ${ }^{2}$ but - intriguingly - a good response to treatment. ${ }^{3}$ In other conditions such as dementia, very early onset may suggest a different disease entity and so direct management accordingly. From the perspectives of surveys, epidemiological research and health service planning, it is also essential to understand the distribution of different disorders over the life course. This underpins precise estimates of projected morbidity and appropriate policy development. For example, most people with chronic psychotic disorders in a mental health service are middle-aged, but our understanding of the onset distribution of these conditions means preventive and early intervention services must target a much earlier age group, as described below.

\section{Defining and measuring age at onset}

This life-course view means that identifying the age at onset of mental disorders is important, but it is difficult. ${ }^{4}$ The classic psychiatric disorders of adult life often begin with non-specific symptoms. As studies of the evolution of symptoms become more detailed, it is clear that early psychopathologic changes can be transient and dynamic before crystallising into a syndrome fitting some operational category such as the American Psychiatric Association's DSM. Only in retrospect can a decision be taken as to whether the early features were in fact part of the eventual picture. In epidemiological terms this leads to enormous scope for error or bias in the recall or memory of events; ${ }^{5}$ some elements may be misattributed to illness and others simply forgotten. From a clinical point of view it is well known that the better the history, the earlier the age at onset can be dated. Furthermore, just as periods of low mood and occasional elation are part of normal life, it seems likely that some psychological experiences such as voice hallucination, hitherto considered psychopathological, are relatively common during certain developmental periods; it is their persistence or coexistence with other features that marks them as significant. This is leading to conceptual difficulties with clinical concepts such as the prodrome that are at best meaningless or confusing when used at the population level, and may be damaging if they lead to interventions that have adverse benefit-to-harm profiles quite different from the clinical situation.

Retrospective accounts of age at onset for some disorders are more useful than others, and perfect for none. Despite the problems alluded to above, it is a reasonable (and useful) assumption that a large proportion of people with psychotic illness eventually receive treatment, especially given that in the definitions of some types of psychosis such as schizophrenia, disability and loss of function are included in their definition. Thus, a survey using retrospective accounts of age at onset for schizophrenia or a broader group of non-affective psychosis yields useful information. It also avoids contamination by the data for the vast majority of young people who may experience psychotic phenomena without ever being ill. However, this is at the cost of excluding the few people with incipient illness but not yet in contact with services and, for many years, of masking researchers to the extent of psychotic phenomena as part of normal experience; for many decades epidemiologists regarded such phenomena simply as false positives. The retrospective approach is also hampered by the long duration of untreated psychosis in many with non-affective psychosis who eventually make service contact, but the alternative approach - using a community survey for incident illness - would be hugely inefficient given the relative rarity of cases and the fact that the illness itself means that affected people would be less likely to take part. ${ }^{6}$ It is important to recognise that the retrospective approach in clinical samples yields a self-fulfilling estimate of the age at onset of psychotic disorders 
that are seen and treated in clinical services, not a broader phenotype that does not meet this threshold. That said, the method - especially when combined with population registers of treated mental disorder - can lead to definitive results. ${ }^{6}$

Using retrospective accounts of age at onset from cohorts of treated individuals is not useful for less disabling mental disorders because most are never treated. In some areas such as cardiovascular disease and cancer it is effective to follow up large cohorts of individuals and track these outcomes which are relatively easy to measure. ${ }^{7}$ This is much more difficult for common mental disorders where the true incidence and age at onset need intensive study, but has been achieved for psychotic illness using population-based birth cohort studies where follow-up is achieved either through regular interviews and surveys, ${ }^{8}$ or through population registers. ${ }^{9}$ Such studies are rare and usually problematic in some way as they were initiated for reasons other than mental health disorders. For these reasons, retrospective reports from carefully designed cross-sectional community surveys provide most evidence on age at onset for most mental health disorders.

Examples of each of these methodological approaches to age at onset are outlined below, taking different exemplar disorders. This is followed by a discussion as to whether the findings on age at onset in mental health disorders fit with what we know about brain development. One final comment about the definition of age at onset arises from modern accounts of many long-term conditions in the framework of life course models. Here, a model of cardiovascular disease in late adulthood might encompass genetic inheritance, the epigenetic modification of the genome by fetal environment and childhood nutrition, the importance of social context in the beginnings of cigarette smoking at school, followed by further genetic and behavioural aspects in the establishment of nicotine dependence and sedentary lifestyle. The example could go on but illustrates the gradual accretion of interacting components to a self-perpetuating cascade of causation. In the domain of mental health disorders the seeds of schizophrenia may be sown in early life, ${ }^{8}$ and early motor developmental abnormalities may betray important information as to the underlying neurobiology of psychotic symptoms in a later phase of development. ${ }^{10}$ However, one would be hard pushed to say that later motor milestones were the beginning of schizophrenia. Thus, in this article, the onset of a disorder is defined as the onset of features that form part of the disorder and that are contiguous with its first expression.

\section{Clinical studies and clinical epidemiology}

\section{Schizophrenia}

Schizophrenia is an archetype among the psychotic disorders for which retrospective accounts reconstructing the evolution of illness in treated clinical samples yield strong results; it is also a disorder with a developmental component. The same arguments also pertain to a broader spectrum of these illnesses seen in clinical practice. ${ }^{11}$ The idea that schizophrenia might be developmental in origin is not new. ${ }^{12}$ It was evident in early clinical descriptions of the syndrome, ${ }^{13,14}$ rediscovered as a concept towards the end of the 20th century, ${ }^{15}$ and received empirical support from epidemiological life course studies shortly afterwards. ${ }^{8}$ However, much of this evidence comes from the early appearance of neurobehavioural and cognitive characteristics that may betray abnormal function relevant to the incipient mental disorder, rather than the clinical entity we call schizophrenia. Large-scale, population-based studies focusing on the onset of schizophrenia itself showed that it appeared characteristically during the post-pubertal epoch. Schizophrenia occurs in childhood but is vanishingly rare before puberty, after which its incidence increases rapidly until the mid-20s before declining over the following decades. This is best illustrated by the seminal studies of Häfner et al in Mannheim, ${ }^{16,17}$ who ascertained all incident cases of schizophrenia occurring in their catchment area and reconstructed the characteristics of their onset in minute detail (Fig. 1). The earliest signs of any mental disturbance occurred before puberty, but psychotic symptoms and index admissions for the full syndrome were confined to the decade and half after puberty. The data for women (Fig. 1(b)) indicate a continued developmental phenomenon into the fifth and sixth decades, where a second, smaller peak in incidence has been linked to changes in oestrogen levels associated with the menopause. These findings have been repeatedly replicated, ${ }^{11}$ and represent some of the most robust findings in psychiatric epidemiology.

\section{Depression and anxiety}

Depression and anxiety, unlike schizophrenia, are well-known entities before puberty. ${ }^{18}$ Follow-up studies of these disorders in children and adolescents in clinical settings indicate a high degree of homotypic continuity into adult life, meaning that the same clinical syndrome is found in both earlier and later life, ${ }^{19-22}$ this being the case also for conduct disorders, which in addition showed a broader range of adverse outcomes (i.e. heterotypic

(a)

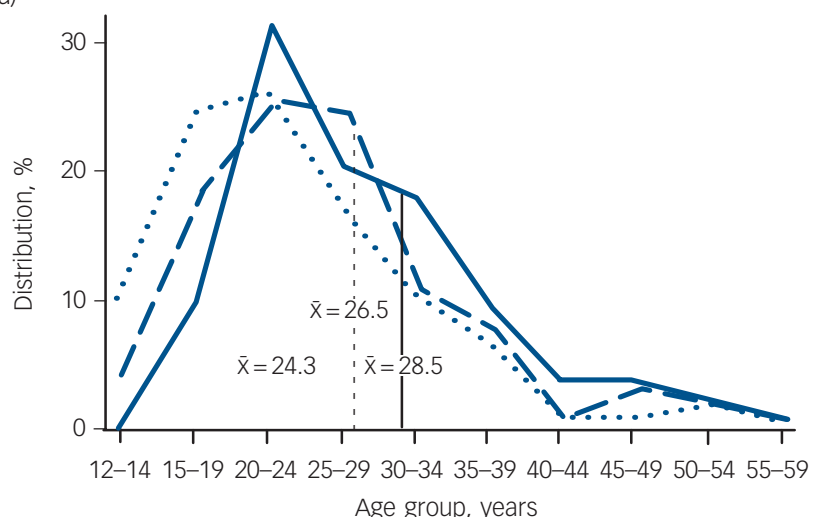

(b)

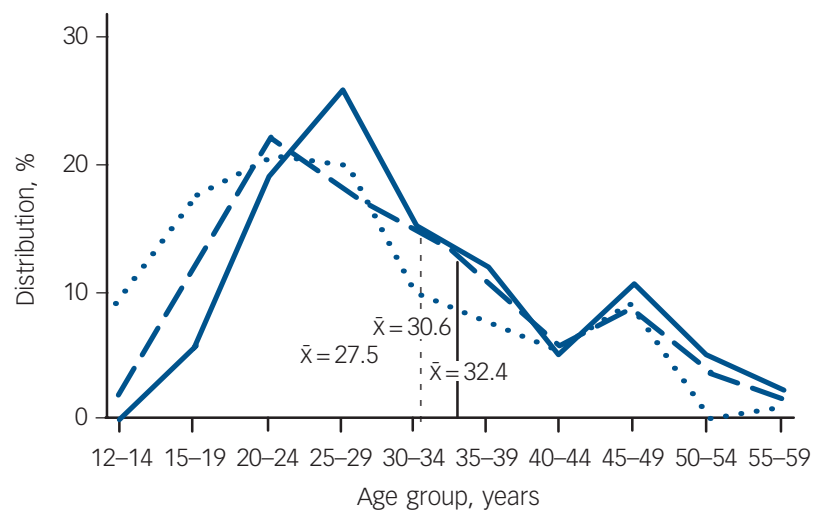

Fig. 1 Distribution of age at onset (as percentage of the whole sample, with being mean age in years) of schizophrenia (broad definition, ICD-9 codes 295, 297, 298.3, 298.4). (a) Males: earliest sign of mental disturbance, $n=117$ (dotted line); first psychotic symptom, $n=125$ (dashed line); index admission, $n=133$ (solid line). (b) Females: earliest sign of mental disturbance, $n=131$ (dotted line); first psychotic symptom, $n=$ (139) (dashed line); index admission, $n=143$ (solid line). From Häfner et al. ${ }^{16,17}$ 
continuity) in addition to homotypic adult antisocial personality traits. $^{23}$ Thus, for the proportion of children and adults who went on to develop the adult syndrome, their adult mental health disorders can be said to have begun in childhood and adolescence.

\section{Population-based approaches}

Children who present to clinical services with psychiatric illness may not be at all typical of those in the general population, where even specific operational diagnoses are common at around one in ten of the population under 16 years old; few of these ever cross the threshold into clinical care. For instance, the British Child and Adolescent Mental Health Survey of 1999 surveyed 10438 children, triangulating information from the children themselves and from their parents and teachers; around 9.5\% had at least one current DSM-IV diagnosis. ${ }^{24}$ Following such large samples into adult life is a considerable undertaking but has been done on several occasions in the context of birth cohorts. In these studies large samples of people defined by geography and birth date or period are followed, often for many years, simply because they represent the general population. Several birth cohorts have contributed a great deal to our knowledge about risk factors and long-term outcomes of psychiatric illness. ${ }^{25}$ Here, we look in more detail at two studies: one from Britain and one from New Zealand. Both studies have been influential in elucidating the link between adult disorders and their adolescent onset.

\section{The British 1946 birth cohort}

Sometimes referred to as the grandfather of birth cohorts, the British 1946 birth cohort is a stratified, random sample of 5362 people born in one week in March 1946, first assessed 6 weeks after birth, ${ }^{26}$ and regularly thereafter, irrespective of their life experiences and masked to their outcomes; these individuals continue to be followed as many contemplate the milestone of retirement and all that is brought by later middle age. Colman et al used ratings made by teachers when the survey members were aged 13 and 15 years (in 1959 and 1962 respectively) that included items akin to those seen in internalising disorders such as depression and anxiety. ${ }^{27}$ Survey participants scoring in the top $6 \%$ of the population distribution were defined as cases of 'common mental disorder' (CMD); 277 were in this group at either age, whereas 46 met case definition at both 13 years and 15 years of age and were defined as 'persistent CMD' cases; interestingly, these observations were made before it was believed that these disorders occurred in children and well before current classifications of mental illness such as DSM and the World Health Organization's (WHO's) ICD. The remaining survey members $(n=3002)$ were classified as healthy, falling into neither group.

The presence of common mental disorder in the teenage years had a profound association with mental health in subsequent surveys of this cohort stretching out into middle life when assessments were made at ages 36, 43 and 53 years. Those in the CMD group were over two-and-a-half times more likely to be rated as having common mental disorder at age 36 years compared with the mentally healthy teenagers, and one-and-a-half times more likely to be ill at age 43 years; the effect was no longer present at age 53 years. For those with persistent CMD as teenagers the outlook was bleaker: they were over six times more likely to be ill with a common mental disorder at ages 36 and 43 years, with the effect persisting at age 53 years, when they were at fourfold risk. The ripples of episodic common mental disorder had faded by middle age but persisted after the age of 50 years for those who had experienced persistent depression and anxiety in their early teenage years; their poor mental health could be traced back to adolescence, perhaps even earlier.

\section{The Dunedin Longitudinal study}

Another example of the birth cohort approach comes from New Zealand and the Dunedin Multidisciplinary Health and Development Study. ${ }^{28}$ This is based on a sample of 1037 children born between April 1972 and March 1973 in Dunedin and first assessed at age 3 years. Now in the era of operational criteria and standardised diagnostic measures, the participants were assessed at ages 11, 13 and 15 years with the Diagnostic Interview Schedule for Children, and then at ages 18, 21 and 26 years with the Diagnostic Interview Schedule; ${ }^{29}$ these measures allowed DSM-IV diagnoses to be generated. ${ }^{30}$ Cohort members receiving treatment and those using intensive mental healthcare were also identified.

The prevalence in the past year of any mental disorder at age 26 years was high at $48 \%$, with men and women being equally affected. Over $80 \%$ of those with a mental health disorder at this age also had a prior diagnosis of any mental illness since the age of 11 years; overall, $74 \%$ had received a diagnosis before 18 years of age and $50 \%$ before 15 years of age. The percentage of prior diagnoses increased according to the severity of disorder by age 26 years in terms of treatment received. Of those with a disorder seeking treatment, $77 \%$ were categorised as meeting diagnostic criteria before 18 years of age and $58 \%$ before age 15 years. For those using intensive mental health services, $80 \%$ received a diagnosis before 18 years of age and $60 \%$ before age 15 years. There was a pattern of homotypic continuity, especially for conduct disorder and adult antisocial personality disorder, but there were also heterotypic associations; adult anxiety and schizophreniform disorders were preceded by a wide range of adolescent diagnoses. For all adult disorders, $25-60 \%$ of cases had a history of conduct and/or oppositional defiant disorder. The authors concluded that the majority of mental health disorders manifest at age 26 years should be considered as extensions of adolescent disorders, just as suggested by Colman et al for common mental disorders in the British 1946 birth cohort. ${ }^{27}$ The eradication of adolescent disorders, particularly conduct disorders, would have a profound effect on reducing subsequent morbidity.

\section{Cross-sectional surveys}

These two birth cohorts give clear evidence that a considerable proportion of mental health disorders are already manifest in adolescence; these studies have many methodological advantages in their prospective approach but are limited by their periodic assessments during the life course, and the relatively small proportion of whole life that they have yet covered. A major advance in our understanding of age at onset distributions has come with developments in the methodology that can be used with cross-sectional mental health surveys. The National Comorbidity Survey Replication (NCS-R) built on a succession of major epidemiological surveys in the USA, particularly the Epidemiological Catchment Area study and the National Comorbidity Survey, and was a major part of a global initiative, the WHO World Mental Health Survey Initiative. ${ }^{29,31-34}$

The NCS-R used the WHO Composite International Diagnostic Interview (WHO-CIDI), ${ }^{35}$ a fully structured lay-administered interview, to generate DSM-IV diagnoses from data collected in a household survey of 9282 respondents (70.9\% response rate). Relative to prior studies, methodological innovations included an expanded set of diagnoses; in-depth clinical validation of field research diagnoses based on clinician-administered Structured Clinical Interview for DSM-IV re-interviews; ${ }^{36}$ dimensional self-ratings on clinically anchored scales; ${ }^{37,38}$ inclusion of subthreshold diagnostic syndromes; assessment of disability and impairment; the use of disease burden metrics 
linked to marker physical disorders; and assessments of service use, treatment barriers and adequacy of treatment. The study was not designed for rare disorders such as schizophrenia and autism which lead to considerable non-response, but generated remarkable information on the lifetime prevalence of many conditions.

A number of methodological enhancements allowed this survey to obtain helpful estimates of age at onset, despite its retrospective method which is often associated with error. ${ }^{5}$ Questions were designed to avoid implausible responses by using a time-line approach, ${ }^{31}$ and by breaking the life course into blocks such as pre-school, high school, university and employment, so that respondents could date both important symptoms (e.g. first panic attack) and full syndromes at least to a certain epoch of their lives. ${ }^{39}$ The ages by which $25 \%, 50 \%$ and $75 \%$ of respondents first became affected are shown for a number of syndrome groups in Table 1 together with their overall lifetime prevalence, standardised to age 75 years.

Overall, there are four headlines from the NCS-R relevant to this review. First, half (51\%) of Americans will experience a DSM-IV mental health disorder during their lifetime, with some evidence that this proportion may be increasing with younger cohorts. Second, half of these disorders (as shown by the 50th percentile or median) were incident by age 14 years. The median onset tended to be earlier for anxiety disorders (age 11 years), some of which begin and end in childhood, and for impulse control disorders (age 11 years) than for substance use disorders (age 20 years) and mood disorders (age 30 years). Third, age at onset was concentrated in a narrow age range for most disorders: interquartile ranges (the number of years of age between the 25th and 75th percentiles of the age-at-onset distributions) were only 8 years (age 7-15 years) for impulse control disorders, 9 years (age 18-27 years) for substance use disorders and 15 years (age 6-21 years) for anxiety disorders, compared with 25 years (age 18-43 years) for mood disorders. Half of all mental health disorders over the life course occurred first between the ages of 7 years and 24 years.

Analysis of service contact data from the NCS-R leads to the fourth major finding and presents a dismal tale of failure, delay and lost opportunity. ${ }^{40}$ The vast majority of people with lifetime disorders eventually made contact with health services, although more commonly for mood disorders (88-94\%) than for anxiety $(27-95 \%)$, impulse control $(34-52 \%)$ or substance use (53-77\%) disorders. Delay among those who eventually made contact ranged from 6 years to 8 years for mood disorders and from 9 years to 23 years for anxiety disorders. Failure to make initial treatment contact at all and delay among those who did eventually make treatment contact were both associated with early age at onset, being older at the time of the survey, and a number of sociodemographic characteristics including being male, married, poorly educated and from a Black and minority ethnic group.

\section{From neurobiology to service provision}

One further question is whether what is happening to the brain during the adolescent period mirrors this tide of incident mental health disorder where, excluding the neurodegenerative disorders of later life, half of all onsets have occurred by the mid-teens.

The brain is constantly changing throughout life; even reading this article may consolidate some aspect of neural circuitry and leave a memory trace. That said, there are several epochs over the life course where there is radical transformation of neural architecture and function. One of these epochs spans the traditional legal, educational and service boundary of 17-18 years of age. In fact, this artificial transition falls right in the middle of a crucial phase of brain development that begins shortly before puberty and ends during the third decade of life. This questions the whole concept of adolescence (based on sexual maturity) being distinct from adulthood (based on a concept of attainment of mental and physical capacity).

Development of the neonatal brain from its ectodermal origins is a dramatic achievement of nature. Itself complex and depending on a variety of processes such as the establishment of connectivity and programmed cell death, followed by a rapid development of basic cabling through myelination in the first year, this epoch ushers in some classical neurodevelopmental disorders and learning delays; but this is only part of the story. It takes three decades to grow a mature human brain, during which time there is much further development. The period from mid-childhood through puberty to the mid-20s is another phase of profound change, particularly in the balance of connectivity between brain areas. ${ }^{41}$ It is during this period that the risk of adult mental health disorders becomes apparent; it may be the characteristics of normal development that lead to this risk, rather than any particular abnormality in this process. ${ }^{42}$ Indeed, aspects of syndromes such as impulse control disorders are so common during adolescence that it is questionable as to whether they should be considered abnormal. This second, more leisurely phase of development has only recently begun to be understood. It is probably heralded by prepubertal maturational events in the adrenal glands known as adrenarche that take place around 6-8 years of age. ${ }^{43}$ Adrenarche involves the production of two steroid compounds, dehydroepiandrosterone (DHEA) and its metabolite dehydroepiandrosterone sulfate (DHEAS), which are abundant within the brain. Longitudinal studies of brain structure using repeated magnetic resonance imaging scans in healthy children have demonstrated considerable pre-adolescent increases in cortical grey matter, peaking some 2-3 years after adrenarche and then declining in an inverted U-shaped manner. ${ }^{44,45}$ Increases

Table 1 Ages at onset for five categories of mental health disorder

\begin{tabular}{|c|c|c|c|c|}
\hline & \multirow[b]{2}{*}{ Projected lifetime risk, ${ }^{a} \%$ (s.e.) } & \multicolumn{3}{|c|}{ Age at which \% of projected lifetime risk attained, years ${ }^{\text {b }}$} \\
\hline & & $25 \%$ & $50 \%$ (median) & $75 \%$ \\
\hline Anxiety disorders & $31.5(1.1)$ & 6 & 11 & 21 \\
\hline Mood disorders & $28.0(0.8)$ & 18 & 30 & 43 \\
\hline Impulse control disorders & $25.4(1.1)$ & 7 & 11 & 15 \\
\hline Substance use disorders & $16.3(0.6)$ & 18 & 20 & 27 \\
\hline Any disorder & $50.8(1.2)$ & 7 & 14 & 24 \\
\hline
\end{tabular}


in white matter occur over a longer period into the third decade. There is a general pattern of childhood peaks of grey matter followed by adolescent declines, functional and structural increases in connectivity and integrative processing; ${ }^{46}$ the balance between limbic and subcortical and frontal lobe functions extends well into young adulthood. ${ }^{46}$ The higher-order association cortices mature after lower-order somatosensory and visual cortices, and phylogenetically older brain areas mature earlier than those more recently evolved such as the frontal cortex. The control of these changes, particularly the initial childhood growth in grey matter, is likely to be dependent on androgens, with regional differences and contrasts between girls and boys being mediated by differences in androgen receptor density and function. ${ }^{47}$ Cortical developments are more dependent on environmental influences and transactions. Although the brains of children and young adults may have similar 'small world' organisation at the global level, they differ significantly in hierarchical organisation and interregional connectivity. These large-scale changes underpin the transition from dependent child to independent adult able to draw on sophisticated learning from reward and punishment, and effective executive and emotional control. ${ }^{48-50}$

Casey et al provide a model of these key brain structural and neurocognitive phenotypes developing at different rates. ${ }^{42}$ They highlight the relevance of normal development in understanding emergence of psychopathology in the developmental epoch from puberty to the late $20 \mathrm{~s} .{ }^{45} \mathrm{~A}$ 'neural maturation gap' during adolescence exists between earlier maturation of limbic and subcortical reward processing networks dependent on the neurosteroid growth spurt, and later consolidation of neocortical cognitive and emotional control networks dependent on environmental experience. This maturation gap may present a window of vulnerability during which these two different systems are not yet fully coordinated.

\section{Health service implications}

It seems that from the perspective of brain development it should come as no surprise that most adult mental health disorders have their roots in adolescence. We may ask why they persist thereafter, and what can be done about them. Mechanisms of persistence are beyond the scope of this article but the robust findings from modern epidemiology and their consilience with the emerging neurobiology of adolescence should be a clarion call for intervention involving both primary and secondary prevention. ${ }^{50}$ Many incident disorders will resolve but it is clear that many do persist, and result in lifelong disability and cost to society and the individual. Not every anxiety disorder or depression in the childhood or teenage years will lead to adult disorder. However, the findings suggest that appropriate interventions should be widely available to speed initial treatment contact and reduce the burdens and hazards of untreated mental disorder. ${ }^{40}$ It is straightforward to propose that early signs of recurrence or persistence of adolescent mental health disorder should lead to a concerted therapeutic effort, probably coordinated by primary care, and there is evidence to suggest that - in psychotic disorders at least - such early specialist intervention is cost-effective. ${ }^{51}$ However, the appropriate response to the earliest signs of disorder in large numbers of young people is less clear, and the risk to benefit ratio of specialist care will be totally different in the wider subclinical or primary care population from that in the services in which many interventions are developed. Traditional mental health services have evolved without the knowledge that psychopathology observes no transition between adolescence and early adulthood. From the perspective of brain development, services may require re-engineering to provide an appropriately seamless and developmentally sensitive approach to individuals on the two-decade journey from puberty to adulthood. These questions are explored elsewhere in this supplement and require considerable and sophisticated research in order to guide appropriate care at the clinical and public health levels.

Establishing age at onset of mental health disorders is methodologically difficult. A range of epidemiological approaches provide convergent evidence not only that adult mental health disorders are common in the population, but that most of them have their onset by adolescence. This period of life after puberty also sees a range of important organisational developments in the brain that last until the late 20s and usher in adulthood as might be defined from a developmental rather than a social or legal point of view. These biological changes are characterised by a temporary uncoupling of higher-order, more recently evolved cortical functions from the limbic and subcortical systems that mature earlier. This maturational gap is normal, but appears to leave the adolescent vulnerable to a range of experiences and responses, some of which form the components of mental disorders and may persist as such. These findings provide a persuasive argument to reappraise our formulations of mental health disorder into a new developmental psychiatry embracing a modern life-course approach that focuses on the second and third decades of life. Such a view also triggers the need for a new research agenda to guide these new ventures, the therapeutic interventions they may wield and the service structures through which these are delivered to young people.

P. B. Jones, PhD, Department of Psychiatry, University of Cambridge, CAMEO Early Intervention Services, Cambridgeshire and Peterborough Foundation National Health Service Trust, Herchel Smith Building for Brain and Mind Science, Cambridge CB2 OSX, UK. Email: pbj21@cam.ac.uk

\section{Funding}

P.B.J. acknowledges research support from the Wellcome Trust and the National Institute for Health Research (NIHR; programme grant RP-PG-0606-1335). The work forms part of the NIHR Collaboration for Leadership in Applied Health Research and Care for Cambridgeshire \& Peterborough.

\section{Acknowledgements}

The author thanks Mrs Anne Robinson and Dr Golam Khandaker for their help with referencing.

\section{References}

1 McGorry P, Johanessen JO, Lewis S, Birchwood M, Malla A, Nordentoft M, et al. Early intervention in psychosis: keeping faith with evidence-based health care. Psychol Med 2010; 40: 399-404.

2 Nicolson R, Lenane M, Hamburger SD, Fernandez T, Bedwell J, Rapoport JL. Lessons from childhood-onset schizophrenia. Brain Res Brain Res Rev 2000; 31: $147-56$.

3 Alvarez-Jimenez M, Gleeson JF, Henry LP, Harrigan SM, Harris MG, Amminger GP, et al. Prediction of a single psychotic episode: a 7.5-year, prospective study in first-episode psychosis. Schizophr Res 2011; 125: 236-46.

4 Kessler RC, Amminger GP, Aguilar-Gaxiola S, Alonso J, Lee S, Ustun TB. Age of onset of mental disorders: a review of recent literature. Curr Opin Psychiatry 2007; 20: 359-64.

5 Simon GE, VonKorff M. Recall of psychiatric history in cross-sectional surveys: implications for epidemiologic research. Epidemiol Rev 1995; 17: 221-7.

6 Perala J, Suvisaari J, Saarni SI, Kuoppasalmi K, Isometsa E, Pirkola S, et al. Lifetime prevalence of psychotic and bipolar I disorders in a general population. Arch Gen Psychiatry 2007; 64: 19-28.

7 Yan LL, Liu K, Daviglus ML, Colangelo LA, Kiefe Cl, Sidney S, et al. Education, 15 -year risk factor progression, and coronary artery calcium in young adulthood and early middle age: the Coronary Artery Risk Development in Young Adults study. JAMA 2006; 295: 1793-800 
8 Jones $\mathrm{P}$, Rodgers B, Murray R, Marmot M. Child development risk factors for adult schizophrenia in the British 1946 birth cohort. Lancet 1994; 344: 1398-402.

9 Isohanni M, Makikyro T, Moring J, Rasanen P, Hakko H, Partanen U, et al. A comparison of clinical and research DSM-III-R diagnoses of schizophrenia in a Finnish national birth cohort. Clinical and research diagnoses of schizophrenia. Soc Psychiatry Psychiatr Epidemiol 1997; 32: 303-8.

10 Ridler K, Veijola JM, Tanskanen P, Miettunen J, Chitnis X, Suckling J, et al. Fronto-cerebellar systems are associated with infant motor and adult executive functions in healthy adults but not in schizophrenia. Proc Natl Acad Sci USA 2006; 103: 15651-6.

11 Kirkbride JB, Fearon $\mathrm{P}$, Morgan C, Dazzan P, Morgan $\mathrm{K}$, Tarrant J, et al. Heterogeneity in incidence rates of schizophrenia and other psychotic syndromes: findings from the 3-center AeSOP study. Arch Gen Psychiatry 2006; 63: 250-8.

12 Crichton-Browne J. The psychical diseases of early life. J Ment Sci 1860; 6: $284-320$.

13 Bleuler E. Dementia Praecox oder Gruppe der Schizophrenien [Dementia Praecox or The Group of Schizophrenias]. Deuticke, 1911.

14 Kraepelin E. Dementia praecox and paraphrenia. In Textbook of Psychiatry, 8th edn (ed. G Robertson, transl. RM Barclay): vol 3, part 2. Livingstone, 1919

15 Murray RM, Lewis SW. Is schizophrenia a neurodevelopmental disorder? BMJ 1987; 295: 681-2.

16 Häfner $\mathrm{H}$, Maurer $\mathrm{K}$, Loffler W, Fatkenheuer B, van der Heiden W, Riecher-Rossler $A$, et al. The epidemiology of early schizophrenia. Influence of age and gender on onset and early course. Br J Psychiatry 1994; 164 (suppl 23): 29-38.

17 Häfner H, Behrens S, de Vry J, Gattaz WF, Löffler W, Maurer K, et al. Warum erkranken Frauen später an Schizophrenie? Nervenheilkunde 1991; 10: 154-63.

18 Goodyer I. The Depressed Child and Adolescent, 2nd edn. Cambridge University Press, 2001.

19 Fombonne E, Wostear G, Cooper V, Harrington R, Rutter M. The Maudsley long-term follow-up of child and adolescent depression: 1. Psychiatric outcomes in adulthood. Br J Psychiatry 2001; 179: 210-7.

20 Harrington R, Bredenkamp D, Groothues C, Rutter M, Fudge H, Pickles A Adult outcomes of childhood and adolescent depression. III. Links with suicidal behaviours. J Child Psychol Psychiatry 1994; 35: 1309-19.

21 Harrington R, Fudge $H$, Rutter M, Pickles A, Hill J. Adult outcomes of childhood and adolescent depression. I. Psychiatric status. Arch Gen Psychiatry 1990; 47: 465-73.

22 Harrington R, Fudge $\mathrm{H}$, Rutter $\mathrm{M}$, Pickles A, Hill J. Adult outcomes of childhood and adolescent depression: II. Links with antisocial disorders. J Am Acad Child Adolesc Psychiatry 1991; 30: 434-9.

23 Fombonne E, Wostear G, Cooper V, Harrington R, Rutter M. The Maudsley long-term follow-up of child and adolescent depression: 2. Suicidality, criminality and social dysfunction in adulthood. Br J Psychiatry 2001; 179: 218-23.

24 Ford T, Goodman R, Meltzer H. The British Child and Adolescent Mental Health Survey 1999: the prevalence of DSM-IV disorders. J Am Acad Child Adolesc Psychiatry 2003; 42: 1203-11.

25 Mann A. The evolving face of psychiatric epidemiology. Br J Psychiatry 1997; 171: 314-8

26 Wadsworth MEJ. The Imprint of Time: Childhood History and Adult Life. Clarendon, 1991

27 Colman I, Wadsworth ME, Croudace TJ, Jones PB. Forty-year psychiatric outcomes following assessment for internalizing disorder in adolescence. Am J Psychiatry 2007; 164: 126-33.

28 Silva PA, Stanton WR. From Child to Adult: The Dunedin Multidisciplinary Health and Development Study. Oxford University Press, 1996.

29 Robins LN, Regier DA. Psychiatric Disorders in America: The Epidemiologic Catchment Area Study. Free Press, 1991.

30 Kim-Cohen J, Caspi A, Moffitt TE, Harrington H, Milne BJ, Poulton R. Prior juvenile diagnoses in adults with mental disorder: developmental follow-back of a prospective-longitudinal cohort. Arch Gen Psychiatry 2003; 60: 709-17.
31 Kessler RC, Berglund P, Demler O, Jin R, Merikangas KR, Walters EE. Lifetime prevalence and age-of-onset distributions of DSM-IV disorders in the National Comorbidity Survey Replication. Arch Gen Psychiatry 2005; 62: 593-602.

32 Kessler RC, Chiu WT, Demler O, Merikangas KR, Walters EE. Prevalence, severity, and comorbidity of 12-month DSM-IV disorders in the National Comorbidity Survey Replication. Arch Gen Psychiatry 2005; 62: 617-27.

33 Kessler RC, McGonagle KA, Zhao S, Nelson CB, Hughes M, Eshleman S, et al. Lifetime and 12-month prevalence of DSM-III-R psychiatric disorders in the United States. Results from the National Comorbidity Survey. Arch Gen Psychiatry 1994; 51: 8-19.

34 Kessler RC, Angermeyer M, Anthony JC, de Graaf R, Demyttenaere K, Gasquet I, et al. Lifetime prevalence and age-of-onset distributions of mental disorders in the World Health Organization's World Mental Health Survey Initiative. World Psychiatry 2007; 6: 168-76.

35 Wittchen HU. Reliability and validity studies of the WHO-Composite International Diagnostic Interview (CIDI): a critical review. J Psychiatr Res 1994; 28: 57-84.

36 Spitzer RL, Williams JB, Gibbon M, First MB. The Structured Clinical Interview for DSM-III-R (SCID). I: History, rationale, and description. Arch Gen Psychiatry 1992; 49: 624-9.

37 Hamilton M. A rating scale for depression. J Neurol Neurosurg Psychiatry 1960; 23: 56-62.

38 Rush AJ, Trivedi MH, Ibrahim HM, Carmody TJ, Arnow B, Klein DN, et al. The 16-Item Quick Inventory of Depressive Symptomatology (QIDS), clinician rating (QIDS-C), and self-report (QIDS-SR): a psychometric evaluation in patients with chronic major depression. Biol Psychiatry 2003; 54: 573-83.

39 Knauper B, Cannell CF, Schwarz N, Bruce ML, Kessler RC. Improving the accuracy of major depression age of onset reports in the US National Comorbidity Survey. Int J Methods Psychiatr Res 1999; 8: 39-48.

40 Wang PS, Berglund P, Olfson M, Pincus HA, Wells KB, Kessler RC. Failure and delay in initial treatment contact after first onset of mental disorders in the National Comorbidity Survey Replication. Arch Gen Psychiatry 2005; 62: 603-13.

41 Blakemore SJ, Burnett S, Dahl RE. The role of puberty in the developing adolescent brain. Hum Brain Mapp 2010; 31: 926-33.

42 Casey BJ, Duhoux S, Malter Cohen M. Adolescence: what do transmission, transition, and translation have to do with it? Neuron 2010; 67: 749-60.

43 Auchus RJ. The physiology and biochemistry of adrenarche. Endocr Dev 2011; 20: 20-7.

44 Giedd JN, Blumenthal J, Jeffries NO, Castellanos FX, Liu H, Zijdenbos A, et al. Brain development during childhood and adolescence: a longitudinal MR study. Nat Neurosci 1999; 2: 861-3

45 Paus T, Zijdenbos A, Worsley K, Collins DL, Blumenthal J, Giedd JN, et al. Structural maturation of neural pathways in children and adolescents: in vivo study. Science 1999; 283: 1908-11.

46 Gogtay N, Giedd JN, Lusk L, Hayashi KM, Greenstein D, Vaituzis AC, et al. Dynamic mapping of human cortical development during childhood through early adulthood. Proc Natl Acad Sci USA 2004; 101: 8174-9.

47 Raznahan A, Lee $Y$, Stidd R, Long R, Greenstein $D$, Clasen L, et al. Longitudinally mapping the influence of sex and androgen signaling on the dynamics of human cortical maturation in adolescence. Proc Natl Acad Sci USA 2010; 107: 16988-93.

48 Galvan A, Hare TA, Parra CE, Penn J, Voss H, Glover G, et al. Earlier development of the accumbens relative to orbitofrontal cortex might underlie risk-taking behavior in adolescents. J Neurosci 2006; 26: 6885-92.

49 Supekar K, Musen M, Menon V. Development of large-scale functional brain networks in children. PLOS Biol 2009; 7: e1000157.

50 Insel TR, Fenton WS. Psychiatric epidemiology: it's not just about counting anymore. Arch Gen Psychiatry 2005; 62: 590-2.

51 Mccrone P, Craig TKJ, Power P, Garety PA. Cost-effectiveness of an early intervention service for people with psychosis. Br J Psychiatry 2010; 196: 377-82. 